\title{
Comparison of dysregulated long noncoding RNAs in lung adenocarcinoma and spinal metastasis: A genome-wide analysis
}

\author{
H. WANG*, S. WANG*, P. LIU, Y. MA, A. HU, C. SUN, J. DONG* \\ Department of Orthopedic Surgery, Zhongshan Hospital, Fudan University, Shanghai, China \\ ${ }^{*}$ Correspondence: dong.jian@zs-hospital.sh.cn \\ ${ }^{*}$ Contributed equally to this work.
}

Received November 11, 2018 / Accepted May 9, 2019

\begin{abstract}
Long noncoding RNAs (lncRNAs) have been shown to play crucial roles in cancer metastasis, yet the lncRNAs landscape of lung adenocarcinoma has not been completely characterized. The aim of this study was to assess the expression profile and potential function of lncRNA in lung adenocarcinoma and in spinal metastasis (SM). A genome-wide microarray analysis was conducted on lung adenocarcinoma and SM tissue from ten Chinese patients. A total of 3,345 differentially expressed lncRNAs were detected. Of those, 761 lncRNAs were upregulated and 2,584 were downregulated (fold-change $>2.0, \mathrm{p}<0.05$ ). These differentially expressed lncRNAs were not evenly distributed among the chromosomes of human genome. Volcano plots of these differentially expressed lncRNAs revealed large variability in lncRNAs expression among 12 patients, indicating that certain lncRNAs may play a positive role in SM of lung adenocarcinoma. Gene Ontology enrichment and pathway analysis identified several remarkably dysregulated biological pathways that affect cell adhesion and the interaction of cytokines and cytokine receptors. Co-expression network analysis showed that 9,458 lncRNAs had verified cis- and trans- target genes. All 2,317 cis targeted genes were confirmed to be differentially expressed and influenced by dysregulated lncRNAs in SM of lung adenocarcinoma. Top ten markedly dysregulated lncRNAs and mRNAs were verified from the co-expression network. In conclusion, this study was a genome-wide survey of dysregulated lncRNAs and corresponding mRNAs that comprise co-regulation networks for SM and lung adenocarcinoma tissues. These dysregulated lncRNAs and mRNA networks could be used as therapeutic gene targets to prevent SM of lung adenocarcinoma and to predictively evaluate treatment efficacy.
\end{abstract}

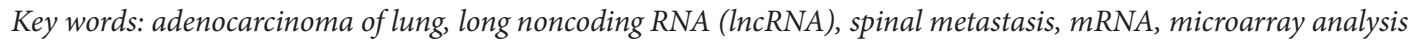

Lung carcinoma is an aggressive and fatal malignancy, which is one of the most common forms of cancer as well as the leading cause of worldwide carcinoma-related deaths [1, 2]. It is a difficult disease to diagnose at an early stage with recently increased morbidity and mortality. This is especially true for spinal metastatic disease (SM) found in 50\% of patients, resulting in paraplegia as well as urinary and fecal incontinence [3]. Twenty-seven percent of patients have rib involvement with appendicular bone involvement in $<6 \%$ of patients $[4,5]$.

Changes in gene expression due to genomic instability, epigenetic alterations and/or chromosome abnormality contribute to lung cancer metastasis [6-8]. However, their role in the pathogenesis of SM remains unclear. In this study genome-wide expression profiling was conducted to assess functionally significant long noncoding RNAs (lncRNAs) and their target genes in SM and lung adenocarcinoma.
LncRNAs are a subset of noncoding RNA transcripts, extensively distributed throughout the genome, that are longer than 200 nucleotides in length [9]. Although the precise function of dysregulated lncRNAs is unclear, they are likely involved in various tumor biological pathways such as cancer glycol-metabolism, apoptosis and cell-cycle progression [10-12]. With the advent of advanced sequencing technology, lncRNAs have been shown to be of diagnostic and prognostic significance in clinical oncology [13, 14], particularly with regard to tumor metastasis $[15,16]$. A few lncRNAs have been shown to be important regulators of lung adenocarcinoma metastasis. For example, HOTAIR and BCYRN1 lncRNAs have been shown to participate in lung cancer development and progression by promoting cell invasion, migration, and metastasis $[17,18]$.

In the present study, we investigated the genome-wide transcriptome profiles of lncRNAs and mRNAs in lung 
adenocarcinoma and SM tissue using next-generation sequencing. We utilized human lncRNA and mRNA arrays to detect the genome-wide transcriptome profile of lung adenocarcinoma tissues and SM tissues from a cohort of patients. Results identified dysregulated lncRNA by quantitative reverse-transcription polymerase chain reaction (qRT-PCR) and their co-expression networks of dysregulated mRNAs. The identified dysregulated lncRNA/mRNA networks may be useful for early detection, prevention and lung adenocarcinoma SM treatment efficacy.

\section{Patients and methods}

Patient recruitment. All patients who did not accept chemotherapy or other forms of treatment were recruited from Zhongshan Hospital Affiliated with Fudan University. Ten tissue specimens were obtained from the Departments of Spinal Surgery and Thoracic Surgery. All tissue specimens were immediately frozen in liquid nitrogen after surgery and stored at $-80^{\circ} \mathrm{C}$. Diagnosis of both lung adenocarcinoma and SM was confirmed pathologically. Clinical data were collected retrospectively from clinical records. This study was approved by the Ethics Committee of the Zhongshan Hospital Affiliated with Fudan University, Shanghai, China. Written consent was obtained from all patients before the surgery.

RNA extraction. Total RNA was extracted from patient samples with Trizol reagent (Life Technologies, USA) following the manufacturer's instructions. The quantity and integrity of the total RNA was tested using the RNA integrity number (RIN) with an Agilent Bioanalyzer 2100 (Agilent Technologies, USA). Quantified total RNA (RIN $\geq 7.0$ and $28 \mathrm{~S} / 18 \mathrm{~S} \geq 0.7$ ) was further purified using a RNeasy mini kit (QIAGEN, GmbH, Germany) and an RNase-Free DNase Set (QIAGEN, GmbH, Germany).

Expression microarray and hybridization. The microarray SBC Human ceRNA microarray v1.0 (Biotechnology Corporation, Shanghai, China) was used, which detected 68,423 lncRNAs and 18,853 mRNAs. RNA samples from each group were then used to generate biotinylated cRNA targets for the SBC human ceRNA array V1.0. Biotinylated cRNA targets were then hybridized to the slides. After hybridization, slides were scanned with an Agilent Microarray Scanner (Agilent Technologies, Santa Clara, CA, US). Microarray experiments were performed by an Agilent Technologies Inc. protocol at Shanghai Biotechnology Corporation.

Gene function analysis. We employed the Database Annotation, Visualization, and Integrated Discovery (DAVID; http://david.abcc.ncifcrf.gov) and implemented Gene Ontology (GO) analysis to identify biological functions of the abnormally expressed genes in the database $[19,20]$. The potential effects of these target genes in tumor pathways were assessed using the Kyoto Encyclopedia of Genes and Genomes (KEGG) (http://www. genome.ad.jp/kegg). Functional pathways were also analyzed by using the Shbio enrichment system [21, 22] (http://enrich.shbio.com). The lower the $\mathrm{p}$-value, the more significant the correlation. The standard cut-off for the p-value was 0.05 .

Long noncoding RNA/mRNA co-expression network. To confirm a lncRNA and mRNA co-expression network, we calculated the lncRNA/mRNA correlation for cis- or trans-regulatory functions. The co-expression network was constructed as described previously. First, genes transcribed within a $10 \mathrm{kbp}$ window upstream or downstream of lncRNAs were considered cis target genes using the genome browser of the University of California, Santa Cruz (UCSC) (http://genome.ucsc.edu). Second, the correlation coefficient was calculated between lncRNAs and mRNAs based on the algorithm of mRNA sequence complementarity and RNA double-chain energy prediction. BLAST software was used for first-round screening, and trans-acting target genes were sought with RNAplex software.

qRT-PCR validation. Total RNA was extracted with Trizol (Invitrogen, CA, USA). The quantity of RNA was assessed with a NanoDrop 1000 spectrophotometer (Thermo Scientific, DE, USA). The ratio of absorbance (A260/A280 $\geq 1.8$ ) was used to evaluate RNA purity. Quantified total RNA was reverse transcribed by a QuantiNovaTM SYBR Green PCR Kit (Qiagen, Hilden, GERMANY). Then, qPCR was conducted with the ABI Prism 7500 sequence detection system (Thermo-ABI 7500, Waltham, MA, USA). Using GAPDH for normalization, expression ratios were calculated using the $2^{-\Delta \Delta C t}$ method. The primers used are listed in Supplementary Table S1. All experiments were repeated three times.

Statistical analysis. SPSS statistical software version 20.0 (SPSS, Woking, UK) and GraphPad Prism 6.0 (GraphPad Software, Inc., CA, USA) were used for statistical analysis. The screening criterion for distinguished lncRNAs and mRNAs was a fold-change $\geq 2.0$ and a threshold p-value $<0.05$. All data are presented as means \pm standard deviation (mean $\pm \mathrm{SD}$ ). The statistical significance of experiments was analyzed by Student's t-test $(\mathrm{p}<0.05)$.

\section{Results}

DE lncRNAs and mRNA in lung adenocarcinoma tissue compared with SM tissue. A total of $68,423 \operatorname{lncRNAs}$ and 18,853 mRNAs were detected as shown in Figure 1. Of the lncRNAs, 3,345 were differentially expressed in SM tissues (fold-change $\geq 2.0, \mathrm{p}<0.05$ ) compared with lung adenocarcinoma in Figure 1A. Among the DE lncRNAs, 761 (22.7\%) were significantly upregulated with fold-change from 2 to 109.9. The top-10 dysregulated lncRNAs are listed in Table 1 and Table 2 .

Of the 18,853 detected by microarray, 1,740 mRNAs were significantly changed (fold-change $\geq 2.0, \mathrm{p}<0.05$ ) in Figure 1B. Of the DE mRNAs, 939 (46.1\%) were upregulated with fold-change from 2 to 1,532.8. The top-10 dysregulated mRNAs are listed in Table 3 and Table 4. 
Table 1. The 10 most upregulated lncRNAs in lung adenocarcinoma tissues compared to spinal metastasis tissues.

\begin{tabular}{|c|c|c|c|c|c|}
\hline Up-lncRNAs & p-values & Fold change & Chromosome & Associated gene & Associated protein name \\
\hline ENST00000518706 & $5.11 \mathrm{E}-05$ & 109.9921 & chr8 & & \\
\hline NR_125830 & $2.79 \mathrm{E}-04$ & 99.4361 & chr8 & & \\
\hline ENST00000609911 & $3.61 \mathrm{E}-05$ & 68.3187 & chr11 & & \\
\hline NONHSAT087996 & $3.07 \mathrm{E}-05$ & 32.5144 & $\operatorname{chr} 3$ & syn3 & synapsin III \\
\hline lnc-BDH2-2:2 & $4.04 \mathrm{E}-06$ & 32.0870 & chr4 & slc9b2 & solute carrier family 9 , subfamily B, member 2 \\
\hline NR_038358 & $2.68 \mathrm{E}-03$ & 22.0184 & $\operatorname{chr} 14$ & & \\
\hline NR_125795 & $1.33 \mathrm{E}-04$ & 21.3160 & chr16 & Foxc2 & forkhead box C2 \\
\hline ENST00000451439 & $3.96 \mathrm{E}-04$ & 16.3333 & chr1 & & \\
\hline lnc-DCAF4L2-3:1 & $4.93 \mathrm{E}-02$ & 15.6970 & chr8 & MMP16 & matrix metallopeptidase 16 \\
\hline lnc-FOXL1-2:4 & $9.62 \mathrm{E}-06$ & 14.3872 & chr16 & & \\
\hline
\end{tabular}

Table 2. The 10 most downregulated lncRNAs in lung adenocarcinoma tissues compared to spinal metastasis tissues.

\begin{tabular}{|c|c|c|c|c|c|}
\hline Down-IncRNAs & p-values & Fold change & Chromosome & Associated gene & Associated protein name \\
\hline lnc-ZNF322-5:1 & $4.84 \mathrm{E}-02$ & 0.0023 & chr6 & & \\
\hline NONHSAT040429 & $6.35 \mathrm{E}-04$ & 0.0084 & - & & \\
\hline NONHSAT072236 & $7.35 \mathrm{E}-04$ & 0.0088 & $\operatorname{chr} 2$ & & \\
\hline NONHSAT040387 & $9.57 \mathrm{E}-04$ & 0.0088 & - & & \\
\hline lnc-D87017.1-2:1 & $8.59 \mathrm{E}-04$ & 0.0113 & $\operatorname{chr} 22$ & Igll1 & immunoglobulin lambda-like polypeptide 1 \\
\hline lnc-RP11-1277H1.1.1-11:1 & $2.91 \mathrm{E}-04$ & 0.0112 & chr16 & & \\
\hline NONHSAT040502 & $1.05 \mathrm{E}-03$ & 0.0116 & chr14 & & \\
\hline NONHSAT072256 & $1.22 \mathrm{E}-03$ & 0.0116 & $\operatorname{chr} 2$ & & \\
\hline NONHSAT072252 & $1.02 \mathrm{E}-03$ & 0.0123 & $\operatorname{chr} 2$ & & \\
\hline lnc-AC110080.1-2:1 & $3.54 \mathrm{E}-03$ & 0.0124 & chr2 & & \\
\hline
\end{tabular}

Table 3. The 10 most upregulated mRNAs in lung adenocarcinoma tissues, compared by volcano plot.

\begin{tabular}{|c|c|c|c|c|c|}
\hline Up-mRNAs & p-values & Fold change & Gene symbol & Chromosome & Description \\
\hline NM_004407 & $4.55 \mathrm{E}-03$ & 1532.8977 & DMP1 & chr4 & dentin matrix acidic phosphoprotein 1 \\
\hline NM_004967 & $3.39 \mathrm{E}-05$ & 1218.5370 & IBSP & $\operatorname{chr} 4$ & integrin-binding sialoprotein \\
\hline NM_001184697 & $9.57 \mathrm{E}-05$ & 429.2515 & MEPE & $\operatorname{chr} 4$ & matrix extracellular phosphoglycoprotein \\
\hline NM_012093 & $1.79 \mathrm{E}-06$ & 124.1880 & AK5 & chr1 & adenylate kinase 5 \\
\hline NM_001044369 & $3.93 \mathrm{E}-05$ & 81.8753 & FAM69C & $\operatorname{chr} 18$ & family with sequence similarity 69 \\
\hline NM_005014 & $4.71 \mathrm{E}-04$ & 81.4318 & OMD & chr9 & osteomodulin \\
\hline NM_001426 & $1.13 \mathrm{E}-03$ & 79.2976 & EN1 & $\operatorname{chr} 2$ & engrailed homeobox 1 \\
\hline NM_053276 & $1.03 \mathrm{E}-03$ & 75.2203 & VIT & chr2 & vitrin \\
\hline NM_001267 & $3.96 \mathrm{E}-02$ & 73.8371 & CHAD & $\operatorname{chr} 17$ & chondroadherin \\
\hline NM_032446 & $6.81 \mathrm{E}-04$ & 62.7354 & MEGF10 & $\operatorname{chr} 5$ & multiple EGF-like-domains 10 \\
\hline
\end{tabular}

Table 4. The 10 most downregulated mRNAs in lung adenocarcinoma tissues, compared by volcano plot.

\begin{tabular}{lccccl}
\hline Down-mRNAs & p-values & Fold change & Gene symbol & Chromosome & Description \\
\hline NM_003357 & $1.35 \mathrm{E}-03$ & 0.0013 & SCGB1A1 & chr11 & secretoglobin family 1A member 1 \\
NM_001172357 & $2.95 \mathrm{E}-03$ & 0.0029 & SFTPC & chr8 & surfactant protein C \\
NM_000192 & $3.47 \mathrm{E}-03$ & 0.0035 & TBX5 & chr12 & T-box 5 \\
NM_152997 & $3.96 \mathrm{E}-03$ & 0.0040 & FDCSP & chr4 & follicular dendritic cell secreted protein \\
NM_207430 & $4.68 \mathrm{E}-03$ & 0.0047 & C11orf88 & chr11 & chromosome 11 open reading frame 88 \\
NM_001198695 & $5.66 \mathrm{E}-03$ & 0.0057 & MFAP4 & chr17 & microfibrillar-associated protein 4 \\
NM_001002919 & $5.89 \mathrm{E}-03$ & 0.0059 & FAM150B & chr2 & family with sequence similarity 150 member B \\
NM_178456 & $7.85 \mathrm{E}-03$ & 0.0079 & C20orf85 & chr20 & chromosome 20 open reading frame 85 \\
NM_007037 & $8.85 \mathrm{E}-03$ & 0.0089 & ADAMTS8 & chr11 & ADAM metallopeptidase with thrombospondin type 1 motif 8 \\
NM_002976 & $9.58 \mathrm{E}-03$ & 0.0096 & SCN7A & chr2 & sodium channel voltage gated type VII alpha subunit
\end{tabular}



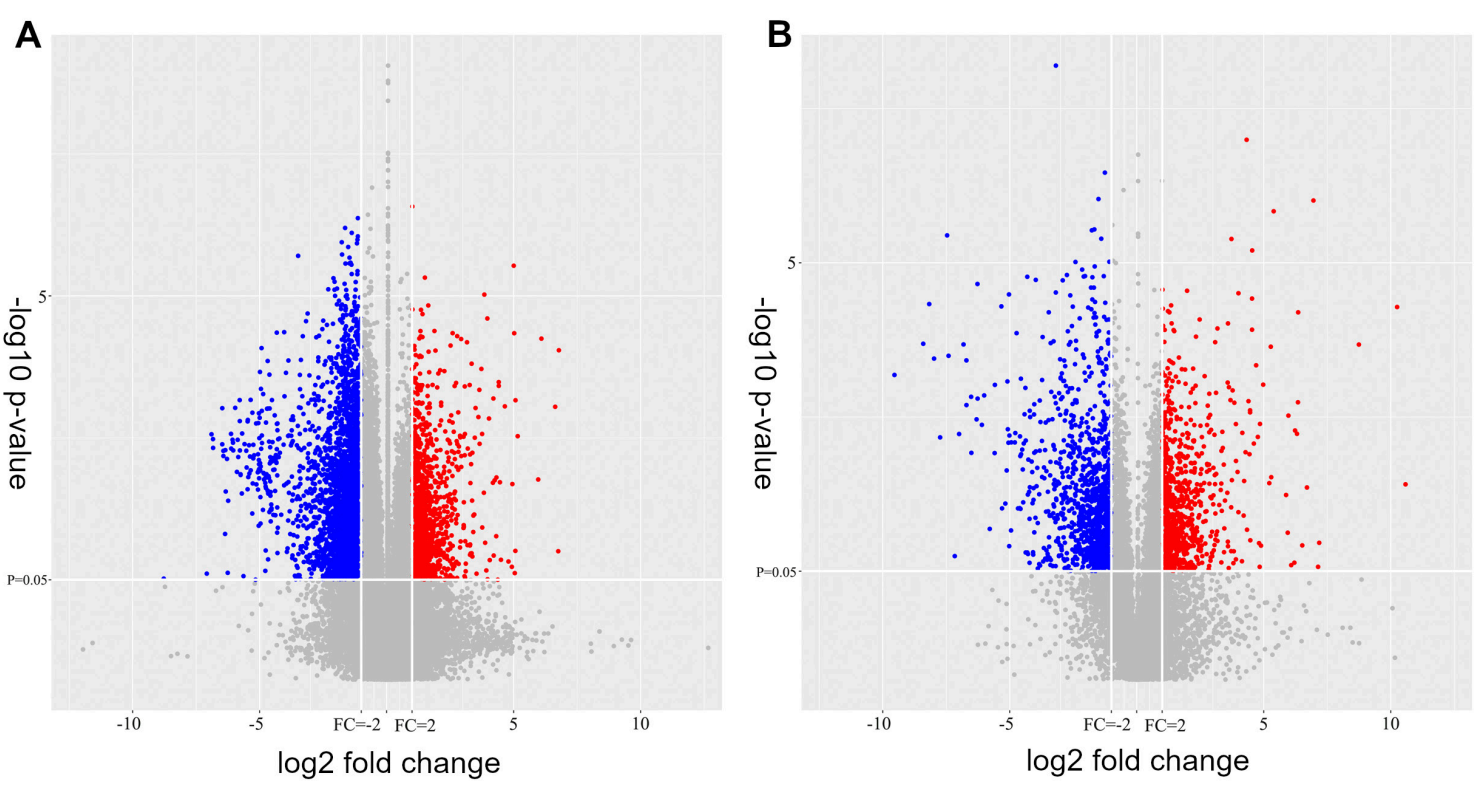

Figure 1. DE IncRNAs and mRNAs in lung adenocarcinoma tissue compared to spinal metastasis tissue, volcano plots of DE IncRNA (A) and mRNA (B). Upregulated and downregulated DE transcripts with fold-change $\geq 2.0$ and $p<0.05$ are shown in red and blue dots, respectively.

A

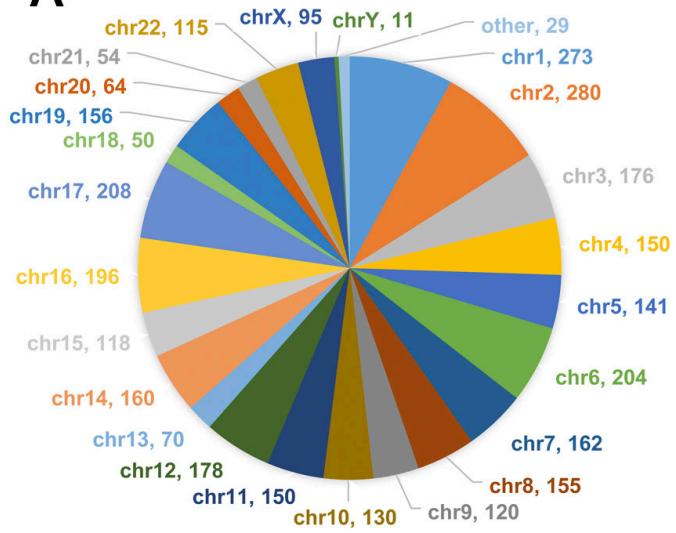

lncRNA

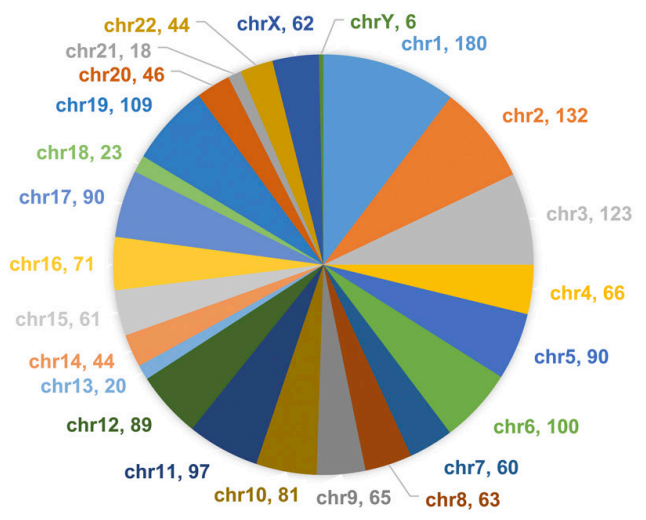

mRNA
B
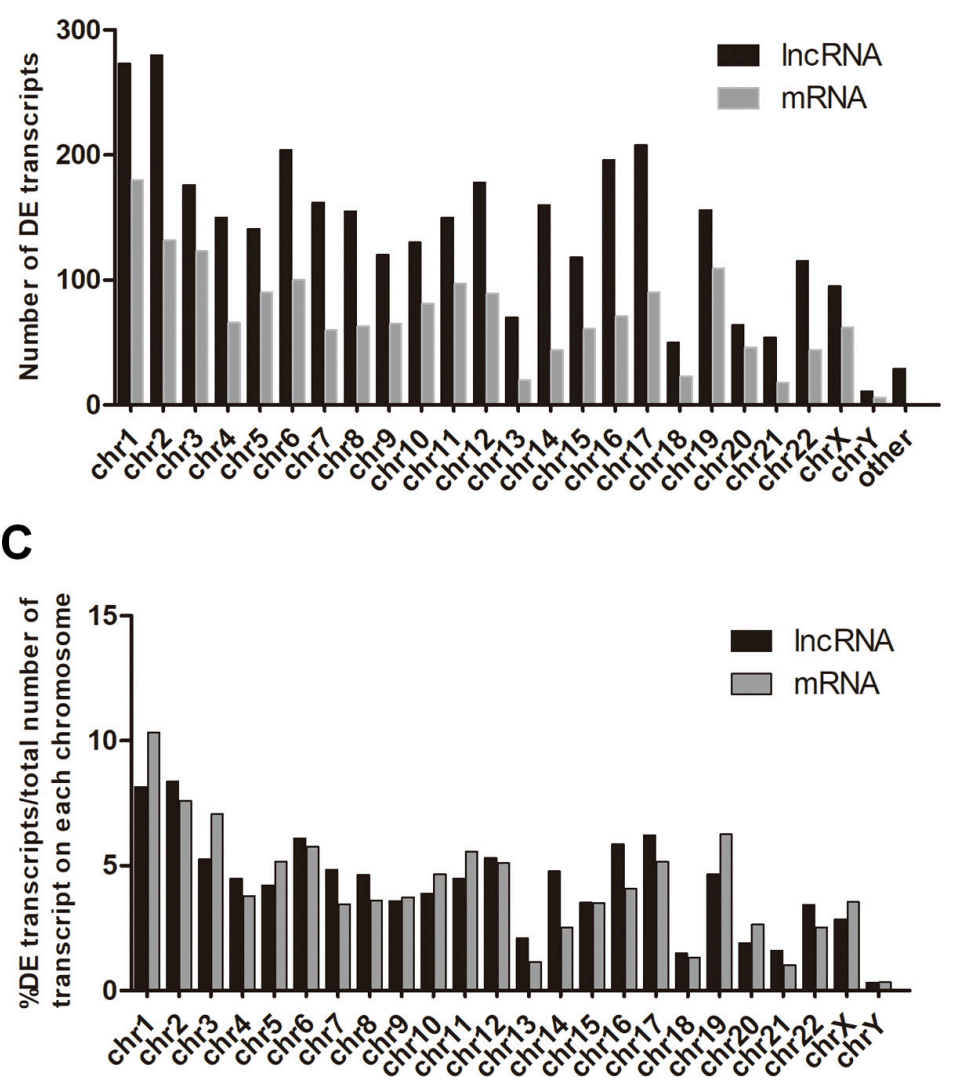

Figure 2. Chromosome distribution of differentially expressed lncRNAs and mRNAs in lung adenocarcinoma tissue compared to spinal metastasis tissue. A) Distribution of differentially expressed lncRNAs and mRNAs on every chromosome. B) Number of differentially expressed lncRNAs and mRNAs on every chromosome. C) Number of differentially expressed transcripts (IncRNAs or mRNAs) on every chromosome. 
Chromosome distribution of DE lncRNAs and mRNAs. Most lncRNAs affect nearby coding genes. Herein, we studied the chromosome distribution of the identified DE lncRNAs and mRNAs within the human genome. These DE lncRNAs and mRNAs were not evenly distributed among the chromosomes (Figures 2A and 2B). The proportional distribution of DE lncRNAs differed among every chromosome (DE lncRNA density), ranging from $0.33 \%$ on chromosome $\mathrm{Y}$ to $8.37 \%$ on chromosome 2 (Figure $2 \mathrm{C}$ ). The percentage of DE mRNAs on each chromosome ranged from $0.33 \%$ on chromosome $\mathrm{Y}$ to $8.37 \%$ on chromosome 1. DE lncRNA density was not consistent with DE mRNA density among the chromosomes. Interestingly, the density of DE lncRNA and DE lncRNA on chromosome $\mathrm{X}$ was higher than that on chromosome $\mathrm{Y}$, suggesting a relative enrichment on chromosome X.

GO enrichment and pathway analysis of DE mRNAs in SM. To explore potential targets of these DE lncRNA in metastatic progression, we implemented GO enrichment and pathway analysis for DE mRNAs (Figure 3). Biological processes, molecular functions and cellular components were detected, and 8,087 were targeted for nearest mRNAs. The top- 5 remarkable GO terms were: urinary bladder development, skeletal muscle satellite cell activation, regulation of natural killer cell mediated immune response to tumor cells, regulation of natural killer cell mediated cytotoxicity directed against tumor cell targets, regulation of myeloid dendritic cell activation.

The Kyoto Encyclopedia of Genes and Genomes (KEGG) pathway analysis disclosed 30 significant KEGG pathways with the threshold (fold-change $\geq 2.0, \mathrm{p}<0.05$ ) (Figure $3 \mathrm{~B}$ ). The top outstanding KEGG pathways were: Viral myocarditis, Type I diabetes mellitus, Toxoplasmosis, $\mathrm{T}$ cell receptor signaling pathway, Synthesis and degradation of ketone bodies. These pathways have been reported to be involved in tumor formation and progression.

Construction of the co-expression network reveals the potential targets (mRNA) of DE IncRNA in SM. To assess biological function, a DE lncRNA/mRNA co-expression network was used to identify possible interactions between mRNAs and lncRNAs. In Figure 4, 1,254 significant connections were found including $298 \mathrm{DE}$ lncRNAs and 127 mRNAs (fold-change $\geq 2.0, \mathrm{p}<0.001$ ). Of the $298 \operatorname{lncRNAs}$ in the co-expression network, the more important networks had more connections, such as lnc-UGDH-1:1 (13 connections), lnc-SMARCA4-2:1 (12 connections), lnc-RNF412:1 (12 connections), lnc-MOK-2:1 (12 connections) and lnc-CXorf26-3:1 (11 connections). The most remarkable mRNAs in the network were marked by the size of the round figure with red color such as: CCL5 (59 connections), 4.3-fold downregulated; TPM3 (52 connections), 4.3-fold downregulated; SERPINB9 (51 connections), 3.7-fold downregulated; RNF207 (46 connections), 2.5-fold downregulated; and CD99 (44 connections), 7-fold upregulated. DE lncRNAs/ mRNAs were densely connected, suggesting their importance as potential targets of mRNA encoded by those IncRNAs; a total of 10 differentially expressed lncRNAs and targeted mRNAs are depicted in Table 5 and Table 6.

The most outstanding and reliably dysregulated lncRNA in all 10 patients was lnc-UGDH-1:1. This downregulated
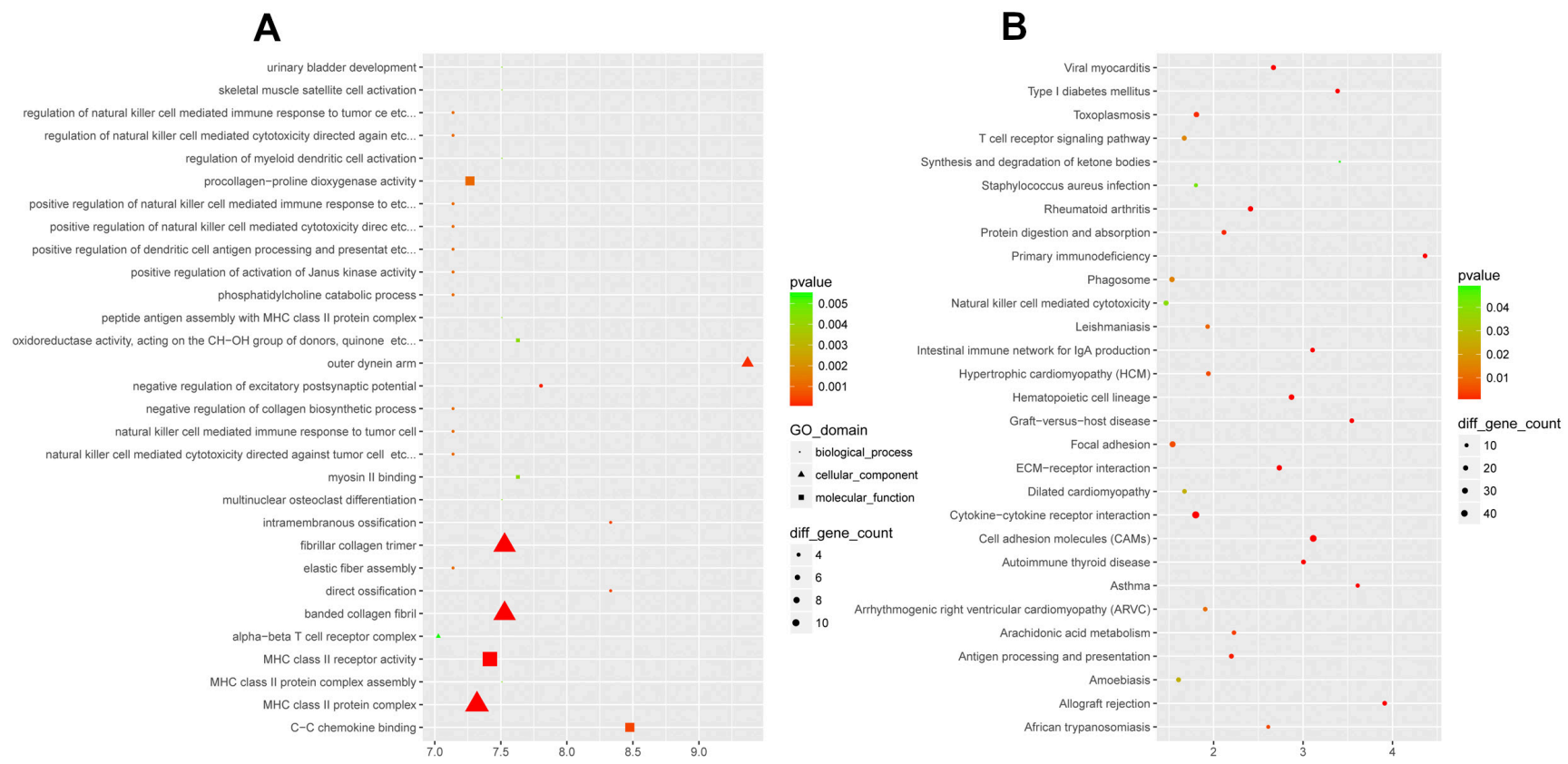

Figure 3. Top 30 GO terms and KEGG pathways significantly enriched in lung adenocarcinoma tissue compared to spinal metastasis tissue. A) Significantly enriched GO terms. B) KEGG pathways of differentially expressed mRNAs. 


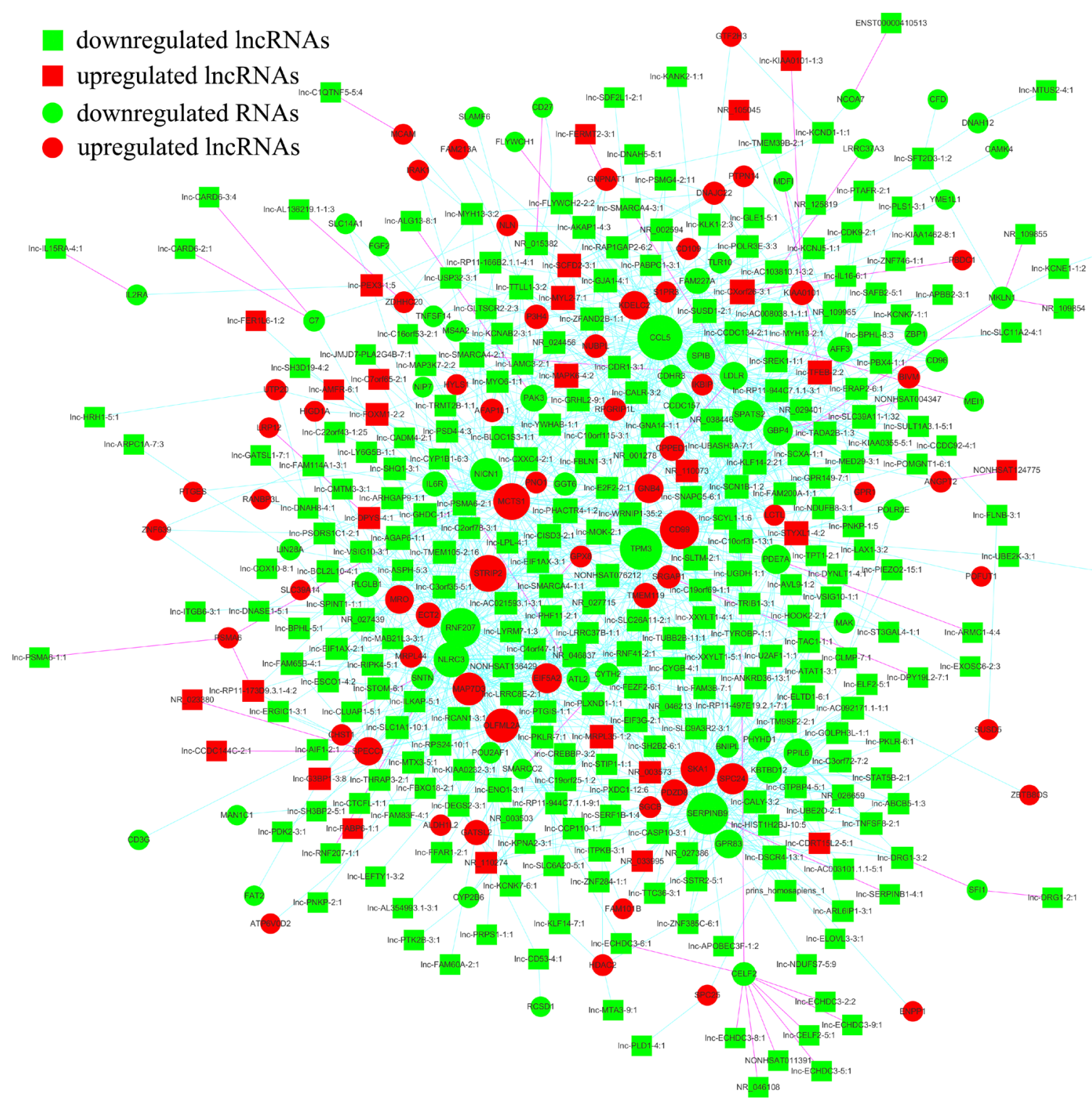

Figure 4. Differentially expressed lncRNA and mRNA co-expression networks in lung adenocarcinoma tissue compared to spinal metastasis tissue. Cis target relations are depicted with red lines and trans target relations with blue lines.

Table 5. A total of 10 cases of dysregulated lncRNAs and nearby coding gene pairs (cis target gene).

\begin{tabular}{|c|c|c|c|c|c|}
\hline \multicolumn{2}{|c|}{ LncRNAs } & \multicolumn{2}{|c|}{ Nearby mRNAs } & \multirow{2}{*}{ Protein name } & \multirow{2}{*}{ Direction(lncRNA-mRNA) } \\
\hline Accession & Fold change & Gene symbol & Fold change & & \\
\hline NONHSAT087996 & 32.5144 & TIMP4 & 25.9989 & TIMP metallopeptidase inhibitor 4 & up-up \\
\hline lnc-DCAF4L2-3:1 & 15.6970 & MMP16 & 5.0451 & matrix metallopeptidase 16 & up-up \\
\hline ENST00000417460 & 10.1667 & TWIST1 & 8.5480 & twist family bHLH transcription factor 1 & up-up \\
\hline lnc-TIMP4-2:1 & 8.2286 & TIMP4 & 25.9989 & TIMP metallopeptidase inhibitor 4 & up-up \\
\hline lnc-AC009336.1-6:1 & 7.0963 & HOXD10 & 2.6528 & homeobox D10 & up-up \\
\hline lnc-HDAC9-8:2 & 6.4170 & TWIST1 & 8.5480 & twist family bHLH transcription factor 1 & up-up \\
\hline ENST00000607019 & 0.3503 & IL16 & 0.2898 & interleukin 16 & up-down \\
\hline NR_038263 & 0.3402 & SOCS2 & 0.3511 & suppressor of cytokine signaling 2 & down-down \\
\hline lnc-WISP2-1:1 & 0.3211 & WISP2 & 0.2567 & WNT1 inducible signaling pathway protein 2 & down-down \\
\hline NONHSAT097315 & 0.2964 & SPARCL1 & 0.3771 & SPARC like 1 & down-down \\
\hline
\end{tabular}


Table 6. A total of 10 dysregulated lncRNAs and their involved coding gene pairs (trans target gene).

\begin{tabular}{|c|c|c|c|c|c|}
\hline \multicolumn{2}{|c|}{ LncRNAs } & \multicolumn{2}{|c|}{ Target mRNAs } & \multirow{2}{*}{ Protein name } & \multirow{2}{*}{ Direction(lncRNA-mRNA) } \\
\hline Accession & Fold change & Gene symbol & Fold change & & \\
\hline lnc-HDAC9-8:2 & 6.4171 & TWIST1 & 8.5480 & twist family bHLH transcription factor 1 & up-up \\
\hline lnc-STYXL1-4:2 & 5.5015 & ANGPT2 & 7.6090 & angiopoietin 2 & up-up \\
\hline lnc-C7orf65-2:1 & 5.3252 & NR4A1 & 0.3466 & nuclear receptor subfamily 4 group A member 1 & up-down \\
\hline lnc-FLYWCH2-2:2 & 0.0751 & KIF14 & 2.1874 & kinesin family member 14 & down-up \\
\hline lnc-CD44-5:1 & 0.1925 & LAMP3 & 0.1903 & lysosomal associated membrane protein 3 & down-down \\
\hline lnc-PKLR-7:1 & 0.1845 & EIF5A2 & 2.5565 & eukaryotic translation initiation factor $5 \mathrm{~A} 2$ & down-up \\
\hline $\operatorname{lnc}-\mathrm{LIN} 28 \mathrm{~B}-5: 1$ & 0.1419 & NR4A1 & 0.3466 & nuclear receptor subfamily 4 group A member 1 & down-down \\
\hline $\operatorname{lnc}-\mathrm{KCNK} 7-6: 1$ & 0.1415 & NR4A1 & 0.3465 & nuclear receptor subfamily 4 group A member 1 & down-down \\
\hline $\operatorname{lnc}-\mathrm{CLMP}-7: 1$ & 0.1294 & TPM3 & 0.3802 & tropomyosin 3 & down-down \\
\hline lnc-CXXC4-2:1 & 0.0894 & TNFSF14 & 0.2536 & tumor necrosis factor superfamily member 14 & down-down \\
\hline
\end{tabular}

lncRNA was associated with 13 mRNAs, 12 of which were also significantly and reliably dysregulated in these samples including: BNIPL (12.5-fold downregulated), CCL5 (4.3-fold downregulated), CD99 (7.2-fold upregulated), GBP4 (2.4-fold downregulated), IRF1 (2.3-fold downregulated), LDLR (3.3-fold downregulated), PPIL6 (4.5-fold downregulated), RNF207 (2.5-fold downregulated), SERPINB9 (3.7-fold downregulated), SPIB (7.4-fold downregulated), STRIP2 (3.3-fold downregulated) and TLR10 (3.8-fold downregulated). Hence, lnc-UGDH-1:1 is an important molecule involved in spinal metastasis by regulation of these target genes.

Experimental validation of DE lncRNAs by qRT-PCR. Top 10 DE lncRNAs and mRNAs (fold-change $\geq 2.0$, $p<0.01$ ) were examined. We performed experimental validation by PCR (Figure 5). We found that 11 ncRNAs and mRNAs expression levels followed microarray data demonstrating a similar trend $(\mathrm{p}<0.05)$.

\section{Discussion}

The significant function of lncRNAs has been widely acknowledged, with recent evidence confirming that lncRNAs play an important role in carcinogenesis and tumor metastasis [23, 24]. Up- and downregulated lncRNAs may be important molecular markers for cancer diagnosis, therapy and tumor metastasis [25-27].

Genome-wide microarray data of the interactions of lncRNAs and mRNAs may also provide for an overall understanding of the mechanism of carcinogenesis. This study is the first to analyze lncRNA profiling and lncRNA/mRNA co-regulation networks for comparison of lung adenocarcinoma and SM, investigating metastasis of lung adenocarcinoma to the spine. Herein, 3,345 differentially expressed lncRNAs were identified with $78.3 \%$ of those downregulated. The majority of DE mRNAs were upregulated in SM tissue compared to lung adenocarcinoma tissue.

Further analysis demonstrated that lncRNA and mRNAs were negatively correlated, suggesting that lncRNAs act
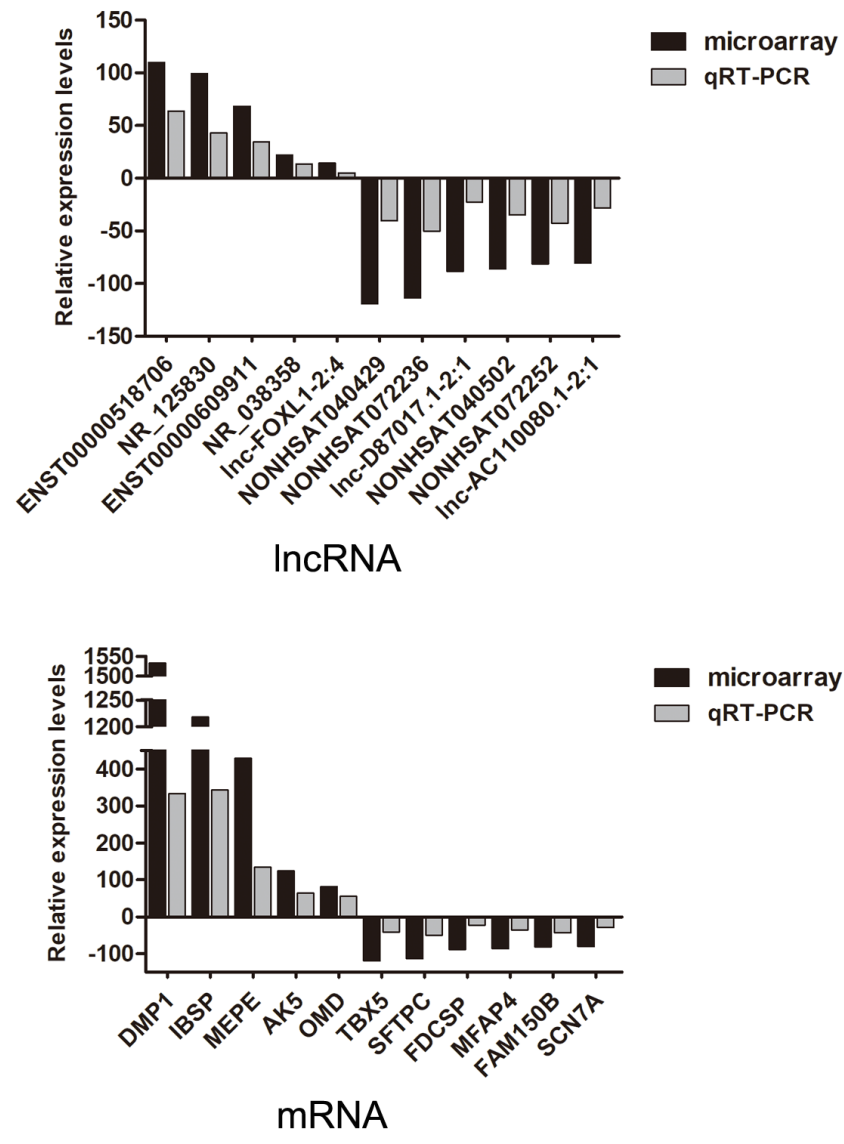

Figure 5. Clinical validation of top 10 differentially expressed lncRNAs and mRNAs by qRT-PCR analysis. Comparison of fold-change for lncRNAs and mRNAs between microarray and qRT-PCR results $(\mathrm{p}<0.05)$.

as negative regulators of coding genes. Gene Ontology and KEGG pathway analysis identified essential biological pathways that underlie lung adenocarcinoma metastasis to the spine. The data reveal that dysregulated mRNAs were enriched in adhesion and cytokine/receptor pathways, which have been reported to be involved in tumor formation and metastasis $[28,29]$. 
Moreover, we further found that differentially expressed lncRNAs/mRNAs were not evenly scattered throughout the human genome. The incidence of lung adenocarcinoma is more common in women than in men [30]. DE transcripts were significantly greater on chromosome $\mathrm{X}$ than chromosome Y, which may relate to increased disease morbidity and mortality among women. There was high variability for dysregulated lncRNAs and mRNAs (fold-change $\geq 2.0$, $\mathrm{p}<0.05$ ), indicating that $\operatorname{lncRNA} / \mathrm{mRNA}$ molecular signatures may facilitate tumor subtype classification. Such a subtype classification would permit a more exact prediction of prognosis and pathology.

One of the most reliably upregulated lncRNAs among the DE lncRNAs was lnc-DCAF4L2-3:1 (chr8,8803201588034562 ) that was as much as 40 -fold upregulated. The coding gene is MMP-16. MMP-16 mRNA was also greatly and reliably upregulated in all SM tissue when compared to lung adenocarcinoma (a fivefold-change). MMP-16 (matrix metalloproteinase 16) is a new member of a family of membrane matrix metalloproteinases that is able to degrade a variety of extracellular matrices as well as to adjust the activity of other family members, promote proliferation, migration and invasion of lung adenocarcinoma cells [31]. Overexpression of MMP-16 may facilitate tumorigenesis and may contribute to tumor cell invasion. Because lnc-DCAF4L2-3:1 was shown to be increased 40-fold, much greater than MMP-16 mRNA (5-fold), lnc-DCAF4L2-3:1 may be a more sensitive biomarker for lung adenocarcinoma metastasis to the spine.

The lncRNA and mRNA co-expression network demonstrated lnc-UGDH-1:1 to be the most significantly downregulated lncRNA and to be the most outstanding with 13 correlated connections. The correlated coding mRNAs included some genes that relate to tumorigenesis and metastasis. For example, CCL5 is a potential pancreatic cancer metastatic marker when combined with CCR5, wherein their interaction increases cancer cell invasion [32]. CD99, a marker of acute myeloid leukemia and myelodysplastic syndromes stem cells can be targeted for therapeutic treatment [33]. Finally, these genes were validated by qRT-PCR. However, the relationship between lnc-UGDH-1:1 and the above potential target genes requires clarification in order to provide a full understanding of the mechanisms of SM of lung adenocarcinoma.

There are limitations to this study: 1) sample size limited examination of DE lncRNAs as biomarkers for metastasis and risk stratification. A larger sample size is required to verify specific $\mathrm{DE} \operatorname{lncRNAs}$ as exact biomarkers; 2) molecular analysis is required to characterize the function of important DE lncRNAs.

In conclusion, genome-wide analysis of DE lncRNAs and lncRNA/mRNA co-expression networks of lung adenocarcinoma and SM revealed a large number of dysregulated IncRNAs and mRNAs, which may serve as biomarkers that guide subtype diagnosis of lung adenocarcinoma, predict prognosis and evaluate metastatic potential.
Supplementary information is available in the online version of the paper.

Acknowledgement: This work was supported by National Natural Science Foundation of China (81572629 and 81772855).

\section{References}

[1] DEVESA SS, BRAY F, VIZCAINO AP, PARKIN DM. International lung cancer trends by histologic type: male:female differences diminishing and adenocarcinoma rates rising Int J Cancer 2005; 117: 294-299. https://doi.org/10.1002/ ijc. 21183

[2] SIEGEL R, NAISHADHAM D, JEMAL A. Cancer statistics, 2013. CA Cancer J Clin 2013; 63: 11-30. https://doi. org/10.3322/caac. 21166

[3] SILVA GT, BERGMANN A, THULER LC. Incidence, associated factors, and survival in metastatic spinal cord compression secondary to lung cancer. Spine J 2015; 15: $1263-$ 1269. https://doi.org/10.1016/j.spinee.2015.02.015

[4] SUGIURA H, YAMADA K, SUGIURA T, HIDA T, MITSUDOMI T. Predictors of survival in patients with bone metastasis of lung cancer Clin Orthop Relat Res 2008; 466: 729-736. https://doi.org/10.1007/s11999-007-0051-0

[5] TSUYA A, KURATA T, TAMURA K, FUKUOKA M. Skeletal metastases in non-small cell lung cancer: a retrospective study. Lung Cancer 2007; 57: 229-232. https://doi. org/10.1016/j.lungcan.2007.03.013

[6] GOTO T, HIROTSU Y, MOCHIZUKI H, NAKAGOMI T, SHIKATA D et al. Mutational analysis of multiple lung cancers: Discrimination between primary and metastatic lung cancers by genomic profile. Oncotarget 2017; 8: 3113331143. https://doi.org/10.18632/oncotarget.16096

[7] GUAN ZB, CAO YS, LI Y, TONG WN, ZHUO AS. Knockdown of lncRNA GHET1 suppresses cell proliferation, invasion and LATS1/YAP pathway in non small cell lung cancer. Cancer Biomark 2018; 21: 557-563. https://doi.org/10.3233/ CBM-170431

[8] ILLEI PB, BELCHIS D, TSENG LH, NGUYEN D, DE MARCHI F et al. Clinical mutational profiling of 1006 lung cancers by next generation sequencing. Oncotarget 2017; 8: 96684-96696. https://doi.org/10.18632/oncotarget.18042

[9] YU AD, WANG Z, MORRIS KV. Long noncoding RNAs: a potent source of regulation in immunity and disease. Immunol Cell Biol 2015; 93: 277-283. https://doi.org/10.1038/ icb.2015.2

[10] DAI W, TIAN Y, JIANG B, CHEN W. Down-regulation of long non-coding RNA AFAP1-AS1 inhibits tumor growth, promotes apoptosis and decreases metastasis in thyroid cancer. Biomed Pharmacother 2018; 99: 191-197. https://doi. org/10.1016/j.biopha.2017.12.105

[11] LI Y, SHEN Z, WANG B, YE C, LAI Z et al. Long non-coding RNA GPR65-1 is up-regulated in gastric cancer and promotes tumor growth through the PTEN-AKT-slug signaling pathway. Cell Cycle 2018; 17: 759-765. https://doi.org/10.10 $80 / 15384101.2018 .1426414$ 
[12] ZENG B, YE H, CHEN J, CHENG D, CAI C et al. LncRNA TUG1 sponges miR-145 to promote cancer progression and regulate glutamine metabolism via Sirt3/GDH axis. Oncotarget 2017; 8: 113650-113661. https://doi.org/10.18632/oncotarget. 21922

[13] ZHOU M, ZHANG Z, ZHAO H, BAO S, CHENG L et al. An Immune-Related Six-lncRNA Signature to Improve Prognosis Prediction of Glioblastoma Multiforme. Mol Neurobiol 2018; 55: 3684-3697. https://doi.org/10.1007/s12035017-0572-9

[14] ZHOU M, ZHAO H, XU W, BAO S, CHENG L et al. Discovery and validation of immune-associated long non-coding RNA biomarkers associated with clinically molecular subtype and prognosis in diffuse large B cell lymphoma. Mol Cancer 2017; 16: 16. https://doi.org/10.1186/s12943-0170580-4

[15] LIN Z, LAI S, HE X, ZHUO W, WANG L et al. Decreased long non-coding RNA MTM contributes to gastric cancer cell migration and invasion via modulating MT1F. Oncotarget 2017; 8: 97371-97383. https://doi.org/10.18632/oncotarget. 22126

[16] XI J, FENG J, ZENG S. Long noncoding RNA lncBRM facilitates the proliferation, migration and invasion of ovarian cancer cells via upregulation of Sox4. Am J Cancer Res 2017; 7: 2180-2189.

[17] GUPTA RA, SHAH N, WANG KC, KIM J, HORLINGS HM et al. Long non-coding RNA HOTAIR reprograms chromatin state to promote cancer metastasis. Nature 2010; 464: 1071-1076. https://doi.org/10.1038/nature08975

[18] LOEWEN G, JAYAWICKRAMARAJAH J, ZHUO Y, SHAN B. Functions of lncRNA HOTAIR in lung cancer. J Hematol Oncol2014; 7:90.https://doi.org/10.1186/s13045-014-0090-4

[19] GENE ONTOLOGY CONSORTIUM. The Gene Ontology (GO) project in 2006. Nucleic Acids Res 2006; 34: D322-326. https://doi.org/10.1093/nar/gkj021

[20] ASHBURNER M, BALL CA, BLAKE JA, BOTSTEIN D, BUTLER $\mathrm{H}$ et al. Gene ontology: tool for the unification of biology. The Gene Ontology Consortium. Nat Genet 2000; 25: 25-29. https://doi.org/10.1038/75556

[21] HUANG DA W, SHERMAN BT, LEMPICKI RA. Bioinformatics enrichment tools: paths toward the comprehensive functional analysis of large gene lists. Nucleic Acids Res 2009; 37: 1-13. https://doi.org/10.1093/nar/gkn923

[22] HUANG DA W, SHERMAN BT, LEMPICKI RA. Systematic and integrative analysis of large gene lists using DAVID bioinformatics resources. Nat Protoc 2009; 4: 44-57. https://doi. org/10.1038/nprot.2008.211
[23] GUTSCHNER T, DIEDERICHS S. The hallmarks of cancer: a long non-coding RNA point of view. RNA Biol 2012; 9: 703-719. https://doi.org/10.4161/rna.20481

[24] MCLEAN GW, CARRAGHER NO, AVIZIENYTE E, EVANS J, BRUNTON VG et al. The role of focal-adhesion kinase in cancer - a new therapeutic opportunity. Nat Rev Cancer 2005; 5: 505-515. https://doi.org/10.1038/nrc1647

[25] LI W, DOU Z, WE S, ZHU Z, PAN D et al. Long noncoding RNA BDNF-AS is associated with clinical outcomes and has functional role in human prostate cancer. Biomed Pharmacother 2018; 102: 1105-1110. https://doi.org/10.1016/j.biopha.2018.03.118

[26] LU C, SHI X, WANG AY, TAO Y, WANG Z et al. RNASeq profiling of circular RNAs in human laryngeal squamous cell carcinomas. Mol Cancer 2018; 17: 86. https://doi. org/10.1186/s12943-018-0833-x

[27] WANG Y, LU Z, WANG N, FENG J, ZHANG J et al. Long noncoding RNA DANCR promotes colorectal cancer proliferation and metastasis via miR-577 sponging. Exp Mol Med 2018; 50: 57. https://doi.org/10.1038/s12276-018-0082-5

[28] CARROLL MJ, FOGG KC, PATEL HA, KRAUSE HB, MANCHA AS et al. Alternatively activated macrophages upregulate mesothelial expression of P-selectin to enhance adhesion of ovarian cancer cells. Cancer Res 2018; 78: 35603573. https://doi.org/10.1158/0008-5472.CAN-17-3341

[29] SAKTHIVEL KM, GURUVAYOORAPPAN C. Targeted inhibition of tumor survival, metastasis and angiogenesis by Acacia ferruginea mediated regulation of VEGF, inflammatory mediators, cytokine profile and inhibition of transcription factor activation. Regul Toxicol Pharmacol 2018; 95: 400-411. https://doi.org/10.1016/j.yrtph.2018.04.012

[30] RODRIGUEZ-LARA V, HERNANDEZ-MARTINEZ JM, ARRIETA O. Influence of estrogen in non-small cell lung cancer and its clinical implications. J Thorac Dis 2018; 10: 482-497. https://doi.org/10.21037/jtd.2017.12.61

[31] YAO Y, SHEN H, ZHOU Y, YANG Z, HU T. MicroRNA-215 suppresses the proliferation, migration and invasion of nonsmall cell lung carcinoma cells through the downregulation of matrix metalloproteinase-16 expression. Exp Ther Med 2018; 15: 3239-3246. https://doi.org/10.3892/etm.2018.5869

[32] SINGH SK, MISHRA MK, ELTOUM IA, BAE S, LILLARD JW JR et al. CCR5/CCL5 axis interaction promotes migratory and invasiveness of pancreatic cancer cells. Sci Rep 2018; 8: 1323. https://doi.org/10.1038/s41598-018-19643-0

[33] TAVAKKOLI M, CHUNG SS, PARK CY. Do preclinical studies suggest that CD99 is a potential therapeutic target in acute myeloid leukemia and the myelodysplastic syndromes? Expert Opin Ther Targets 2018; 22: 381-383. https://doi.org/ $10.1080 / 14728222.2018 .1464140$ 